\title{
Leiomiosarcoma paratesticular de larga evolución. Revisión conceptual y de la literatura
}

\author{
Ruiz Liso JM, Ruiz García J**, Pardo López ML, Vaillo Vinagre A, Gutiérrez Martín A, \\ Bermúdez Villaverde R*.
}

\begin{abstract}
Servicio de Patología/*Servicio de Urología. Complejo Hospitalario de Soria. Hospital Virgen del Mirón. Soria. **Hospital Gregorio Marañón. Madrid.
\end{abstract}

Actas Urol Esp. 2008;32(7):727-736

\section{RESUMEN}

LEIOMIOSARCOMA PARATESTICULAR DE LARGA EVOLUCIÓN. REVISIÓN CONCEPTUAL Y DE LA LITERATURA Introducción: Conocer la incidencia y características clínicopatológicas del leiomiosarcoma (LMS) paratesticular con la aportación de un caso de larga evolución, estudiando los criterios histopatológicos que deben valorarse en este tipo de neoplasias.

Material y métodos: Se incluye para estudio un paciente de 73 años con tumoración escrotal izquierda presente desde hace 30 meses. Se realiza estudio histopatológico e inmunohistoquímico. De igual forma se realiza un examen bibliográfico de este tipo de tumores y sus diferentes subtipos a través de Medline y de búsqueda no indexada.

Resultados: Nuestro caso es el segundo de mayor evolución de la literatura internacional, el primero en mayores de 60 años y uno de los de menor índice mitótico ( 1 x 50 cga). Sin embargo, su atipia, necrosis tumoral próxima al $50 \%$ de la lesión y su pleomorfismo, así como su inmunofenotipo se corresponde con un LMS bien diferenciado grado 1 (French Federation Cancer).

Hemos encontrado tan solo 107 casos publicados de este tipo de sarcomas, de ellos 5 en nuestro país, lo que da un perfil de la baja incidencia de estas neoplasias, que dentro de los sarcomas no alcanzan el 1\%. Incluimos el perfil clínicopatológico de los mismos.

Conclusiones: La larga evolución de una tumoración paratesticular y la ausencia de metástasis no excluye su malignidad. La baja incidencia de los LMSs en esta localización implica que no exista un protocolo de actuación consensuado para los mismos. Consideramos de gran importancia su creación por parte de urólogos, oncólogos, radiólogos y patólogos al igual que se hizo con los localizados en retroperitoneo y tejidos blandos profundos.

Palabras clave: Leiomiosarcoma. Paratesticular. Evolución. Cordón espermático.

\section{ABSTRACT}

LONG-TERM PARATESTICULAR LEIOMIOSARCOMA. CONCEPTUAL AND LITERATURE REVIEW

Introduction: To know the incidence and clinical and pathologic characteristics of the paratesticular leiomyosarcoma (LMS) with the contribution of a case of long evolution, studying the histopathologic criteria that must be valued for this type of tumours.

Material and metods: A 73-year-old patient includes for study with scrotal tumour present left side for 30 months. Histopathologic and imunohistochemical study is realized. Of equal form there is realized a bibliographical search of this type of tumours and their different subtypes across Medline and of not index-linked search.

Results: Our case is the second of greater evolution of international literature, first in greater of 60 years and one of those of smaller mitotic index ( 1 x 50 cga). Nevertheless, his atipia, tumorlike necrosis next to $50 \%$ of the injury and its pleomorphic properties, as well as his inmunophenotypic characterization corresponds with a well differentiated LMS, degree 1 (French Federation Cancer).

We have found only 107 published cases of this type of sarcomas, of them 5 in our country, which gives to a profile of the low incidence of these tumours, that within sarcomas $1 \%$ do not reach. We included the clinical and pathologic profile of such.

Conclusions: The long evolution of a paratesticular tumour and the absence of metastasis does not exclude its malignancy. The low incident of the LMSs in this location, implies that a protocol of actuation validate for such does not exist. We considered of great importance, its creation on the part of urologist, oncologists, radiologists and pathologists like he took control of located in retroperitoneal cavity and deep soft tissues.

Keywords: Leiomyosarcoma. Paratesticular. Long-term. Spermatic cord. 
$\mathrm{L}$ os tumores del cordón espermático y de la región paratesticular son raros y como tales su verdadera incidencia no ha sido nunca establecida.

El área anatómica del parateste es compleja. Contiene el conducto deferente, las túnicas vaginales testiculares, epidídimo y restos vestigiales así como los testículos. Histogenéticamente, esta zona está compuesta de varias estructuras tisulares: epiteliales, mesoteliales y mesodérmicas. Por este motivo, los patrones tumorales que surgen en esta región son heterogéneos y con diferente biología (Tabla 1). Clínicamente los tumores paratesticulares son indistinguibles de los tumores testiculares, lo que provoca que inicialmente se presten a un diagnóstico, en muchos casos, erróneo. La mayoría se presentan como una masa escrotal o bien como una tumefacción que puede o no ser dolorosa y ocasionalmente acompañarse de un hidrocele. No suele distinguirse preoperatoriamente entre lesiones benignas y malignas paratesticulares, lo que incide en un problema sobreañadido ${ }^{1,2}$. Aunque infrecuentes, tienen una significativa tasa de malignidad, próxima al 30\% $\%^{1,2}$. Cerca del 50\% de estos tumores recurren a nivel locoregional y las metástasis a veces aparecen incluso 15 años después de la extirpación de la lesión primaria ${ }^{3,4}$. Señalar, también, que la incidencia de las lesiones paratesticulares de

Tabla 1. Tumores del escroto intra y paratesticular (OMS) ${ }^{2}$

- Lipoma
- Linfangioma
- Hemangioma
- Leiomioma
- Neurofibroma
- Schwannoma
- Tumor de células granulares
- Angiofibroma celular: tumor tipo angiomiofibro-
blastoma del varón
- Pseudotumor fibroso calcificante
- Hamartoma fibroso de la Infancia
- Liposarcoma
- Leiomiosarcoma
- Fibrohistiocitoma Maligno
- Fibrosarcoma
- Rabdomiosarcoma

*A ellos deberíamos añadir el Tumor desmoplásico de células redondas ${ }^{1}$. partes blandas es difícil de estimar, especialmente en el caso de los tumores benignos, que por lo general no suelen ser publicados ${ }^{5}$. Lo que es evidente es que la mayoría de los tumores malignos paratesticulares son sarcomas. Significar, también, que la presencia de hidrocele en asociación con un tumor paratesticular es generalmente un signo de malignidad ${ }^{2,4,6}$.

El 0,8-1\% de los sarcomas de partes blandas del adulto son del área paratesticular y de ellos el 30\% son leiomiosarcomas (LMSs). El cordón espermático es la afectación más frecuente, y le corresponden de igual forma el 30\% aproximadamente de todos los sarcomas genitourinarios ${ }^{7-22}$.

Las series más grandes de sarcomas del área paratesticular, muestran una incidencia desigual, según las diferentes publicaciones, siendo los de mayor frecuencia los liposarcomas, seguidos de los LMSs y rabdomiosarcomas (Tabla 2).

En este artículo revisamos las características de los LMSs paratesticulares publicados hasta la fecha, aportando un nuevo caso con un patrón clínicopatológico infrecuente, valorando la necesidad de un protocolo específico para los sarcomas de esta localización.

\section{MATERIAL Y MÉTODO}

\section{Revisión de la Literatura}

Revisada la literatura científica a través de Medline y búsqueda no indexada, hemos encontrado hasta el mes de Agosto de 2007 un total tan solo de 107 casos publicados (completadas a través de otros sistemas de búsqueda), de LMSs del cordón espermático y áreas paratesticulares. Nuestro caso es, pues, el número 108 de las series tras su revisión y reevaluación histológica e inmunohistoquímica ${ }^{1-16,23-84}$.

\section{Revisión Conceptual}

Durante muchos años, el LMS se ha valorado como el sarcoma más frecuente en adultos con un intervalo amplio del 31,5\% sobre más de 8.200 sarcomas al $45 \%$ de todos los tumores malignos de partes blandas, según las series. También hay amplia variación en su localización: 30,7\% a 70\% surgen en extremidades y de un 11,7 a 16\% en retroperitoneo, siendo infrecuentes en la región inguinal ${ }^{1,8,9,14-}$ 19,23,24,27-30.

Son tumores que se presentan en la edad adulta y con las características clinicopatológicas que describimos en la Tabla 3. 
Tabla 2. Sarcomas paratesticulares. Series porcentuales ${ }^{1,4}$, 11, 15, 16, 19, 30-33, 38

\begin{tabular}{|c|c|c|c|c|c|c|}
\hline Autor & Total & Leiomiosarcoma & Liposarcoma & FHM & Rabdomiosarcoma & Otros \\
\hline Khoubehi B - & Rev. & $32 \%$ & $20 \%$ & $13 \%$ & $24 \%$ & Sd \\
\hline Dotan & 57 & $19 \%$ & $44 \%$ & Sd & $19 \%$ & $18 \%$ \\
\hline Coleman & 47 & $19 \%$ & $51 \%$ & $11 \%$ & $13 \%$ & Sd \\
\hline Rao & 8 & $38 \%$ & $24 \%$ & -- & $38 \%$ & - \\
\hline Russo & 14 & $29 \%$ & $21 \%$ & $7 \%$ & $36 \%$ & $7 \%$ \\
\hline Ballo & 32 & $19 \%$ & $25 \%$ & $37 \%$ & Sd & $19 \%$ \\
\hline Berkmen & 17 & $24 \%$ & $31 \%$ & - & $45 \%$ & - \\
\hline Fagundes & 18 & $39 \%$ & $39 \%$ & $11 \%$ & - & $11 \%$ \\
\hline Merimski & 16 & Sd & $\mathrm{Sd}$ & Sd & $\mathrm{Sd}$ & Sd \\
\hline Catton - & 21 & Sd & $\mathrm{Sd}$ & Sd & Sd & Sd \\
\hline Jo- & $\mathrm{Sd}$ & $10 \%$ & $\mathrm{Sd}$ & $\mathrm{Sd}$ & $\mathrm{Sd}$ & $\mathrm{Sd}$ \\
\hline
\end{tabular}

Sd $=$ sin datos.

Tabla 3. Características clinicopatológicas de los leiomiosarcomas paratesticulares ${ }^{1-16,22-84,93}$

- Edad Media de Presentación: 56 años [intervalo etario 16-91 años]

- Ausencia de Metástasis en el acto diagnóstico: 83\% [intervalo 71-92\%]

- Tamaño: <5 cm:39\%; 5-10 cm: 45\%; > 10 cm:16\%

- La mayoría se presentan como masas solitarias únicas o lobuladas.

- Factores Pronósticos Negativos:

- Alto grado de malignidad histológica

- Metástasis en el momento diagnóstico

- Ausencia de subtipos histológicos

- Tamaño tumoral grande. Mayores de $10 \mathrm{~cm}$

- Resección incompleta con márgenes positivos

En los adultos, se presentan como masas nodulares discretas, frecuentemente próximas o surgiendo del cordón espermático y menos a menudo en el epidídimo, con neta separación del parénquima testicular, palpables en el canal inguinal o/y en el escroto, que antes de cualquier intervención quirúrgica deberían ser evaluadas por ecografía escrotal. Si la biopsia intraoperatoria confirma el origen sarcomatoso o maligno de la lesión procede realizar una orquiectomía radical con ligadura alta del cordón espermático y escisión de todos los tejidos blandos periféricos.

Los riesgos de recurrencia local son elevados (50-60\%), tras la aparente "escisión completa" y con mayor potencial de agresividad que en la lesión primitiva.
Los datos histológicos son escasos en una gran parte de las publicaciones y ausentes en muchas de las series revisadas. Además, el contraste diagnóstico ha sido realizado por algunos autores con resultados muy decepcionantes. Hay investigadores que han reclasificado los sarcomas de partes blandas de sus registros, ya que la inmunohistoquímica ha permitido conocer sus fenotipos, observando a nivel de la región paratesticular el hecho de que el 35\% de los mismos estaban mal diagnosticados (periodo 1958-1967) ${ }^{8}$. Así en una revisión de 130 sarcomas de partes blandas, en el caso de los fibrohistiocitomas malignos (FHMs), hubo de realizarse una reevaluación de los criterios histológicos, ya que la validez del diagnóstico del tipo histológico entre 4 patólogos, de reconocido prestigio, tuvo una sensibilidad del 73-75\% y una especificidad del $93-95 \%{ }^{60}$. En este sentido, han sido diagnosticados como LMSs algunos liposarcomas con diferenciación leiomiomatosa así como FHM. Hay casos muy minoritarios con diferenciación mixoide, epitelioide y pleomórficos con células gigantes multinucleadas, siendo la mayoría sin embargo de patrón fascicular clásico. De igual forma se describen casos de lipo-leiomiosarcomas (sarcomas mixtos) ${ }^{81}$ como casos aislados en la bibliografía internacional.

Un hecho relevante es el hallazgo de un caso con LMSs sincrónicos $(\dot{)}$ ) en ambos testículos con un intervalo de presentación corto $(12 \text { meses })^{54}$. La mayoría de los localizados en esta región son LMSs de bajo grado de malignidad (Fisher C French 1) y así lo ponen de manifiesto las revisiones efectuadas, con marcados índices de supervivencia y baja actividad metastásica. No obstante, en el estudio efec- 
tuado hemos hallado tumores metastásicos en toda la economía humana.: cerebro, pulmón, piel, órbita ocular, hueso y viscerales.

\section{Inmunofenotipo}

Coindre J.M ${ }^{8}$ en el estudio inmunohistoquímico (IHQ) para el diagnóstico de los tumores de partes blandas que realizó en el año 2003, puso de manifiesto, de una forma taxativa, que los FHMs no tienen marcadores específicos, pero que la inmunohistoquímica debe ser usada como norma para su diagnóstico diferencial con otros tumores, sean tumores malignos mesenquimales o no, con una línea específica diferenciadora. Para él, la IHQ es decisiva en los sarcomas paratesticulares en casos de rabdomiosarcomas y tumores vasculares. En los casos de LMSs y liposarcomas son inconstantes e inespecíficos y deben ser valorados con precaución. Ya Miettinem en 1990, había enfatizado esta misma opinión ${ }^{85}$.

Los marcadores para músculo liso y otras estirpes mesenquimales son positivos. Los LMSs son negativos para citoqueratinas, presentando en general expresión positiva para vimentina y alfa-1-antiquimotripsina, y focalmente reactiva para CD-68. Algunos tumores han mostrado también positividad para enolasa neuroespecífica y proteína S-100 ${ }^{84}$. Son también invariablemente negativos para marcadores hematolinfoides, hecho este a significar, dado que el dismorfismo de las células semejando células de Reed Sternberg y el componente necrótico-inflamatorio pueden sugerir un proceso linfoproliferativo hodgkiniano tipo celularidad mixta o bien uno no hodgkiniano ${ }^{84-89}$.

En la revisión bibliográfica también hemos encontrado tumores escrotales de patrón histológico inusual (fibrohistiocitoma atípico) tanto por su localización como por su inmunofenotipo, con expresión positiva para músculo liso y c-kit (CD117) ${ }^{84,90}$.

En relación con posible componente hormonal, hemos hallado 2 casos con cifras elevadas de betaHCG en suero y otro caso con elevación de feto proteína, básica, que han sugerido a los autores, síndromes paraneoplásicos ${ }^{78,79,83}$.

Asimismo, como curiosidad, la práctica totalidad de los LMSs se centran en la raza blanca y amarilla con un número importante, superior al 35\%, en japoneses $\mathrm{y}$ un caso intratesticular en un hombre de raza negra.

Algunos autores establecieron una relación entre la inmunoreactividad positiva para p-53 con un peor pronóstico, independientemente del grado tumoral, mostrando un espectro semejante al de los carcino- mas $^{91}$. Otros, no justifican esta relación, considerando más acertado la sobre expresión de Ki-67 ligada a otros factores pronósticos y siendo para ellos el tamaño tumoral y el grado histológico los principales factores pronósticos. Ahlen et al. en el año 2003, descartan a su vez la implicación pronóstica de p-53, Ki-67 y p27, postulando sin embargo, una relación entre una alta expresión de Bcl-2 y el riesgo de desarrollar metástasis y/o recurrencias ${ }^{91-93}$.

Por otro lado, es importante significar el trabajo de Potti en 2004, en el que observa en un estudio de 273 sarcomas de partes blandas que la sobre-expresión del marcador c-kit (CD 117) y del factor de crecimiento (VEGF) en FHMs incrementa su supervivencia, mientras que la sobre-expresión de VEFG la disminuye en el caso de los LMSs, con significación estadística $\mathrm{p}=0,03^{88}$.

La investigación con nuevos marcadores se orienta en la actualidad en la evaluación de mecanismos autocrinos/paracrinos de control de proliferación en estos tumores. La angiogénesis de los FHMs muestra junto con los liposarcomas una mayor densidad de neovasos por unidad de área, que el resto de los sarcomas de partes blandas, lo que podría postular una terapia angiogenética complementaria a los métodos tradicionales ${ }^{94}$.

\section{CASO CLÍNICO}

Paciente varón de 73 años, con tumoración escrotal izquierda de más de 2 años de evolución, doloroso a la palpación. No fiebre ni síndrome miccional. Ausencia de adenopatías inguinales. Teste derecho normal. Analítica: alfa fetoproteína, beta HCG y LDH en rangos normales.

La ecografía escrotal muestra una masa sólida de ecoestructura abigarrada y multinodular de testículo izquierdo con mínimo hidrocele.

El TAC abdomino-pélvico, posterior a la orquiectomía radical izquierda para estudio de extensión, no muestra patología tumoral, locoregional ni a distancia. 6 meses después de su intervención, el paciente está libre de recidivas y no muestra ningún efecto funcional ni orgánico de su proceso.

En el servicio de Patología de nuestro Complejo Hospitalario recibimos una pieza de orquiectomía que mide $13 \times 8 \times 6 \mathrm{~cm}$, mostrando una superficie externa lisa, brillante, sin signos infiltrativos. Al corte, observamos un hidrocele de $5 \mathrm{~cm}$ de diámetro, rodeando un parénquima testicular conservado, sin infiltración neoplásica. Sobre él y sin contacto con el mismo, surge 
una tumoración amarillenta, polilobulada, pseudoencapsulada que aparece entre vaginal y epidídimo, englobando cordón espermático, con zonas amplias de necrosis y hemorragia. El tamaño de esta neoformación es de 6,5 x 6,5 x 4,8 cm. El extremo de resección, vasos y tejido fibroconectivo periférico están libres de tumoración, sin infiltración peritesticular. (Fig. 1)

Microscópicamente, encontramos una neoformación mesodérmica que forma estructuras fasciculares entrecruzadas entre sí, de citoplasmas eosinófilos con bordes poco netos y núcleos hipercromáticos de cromatina periférica densa con marcada anisocitosis y anisocariosis, núcleos muy dismórficos con vacuolización en muchos de ellos, algunos polilobulados coexistiendo con otras células atípicas multinucleadas en menor cuantía. Amplias zonas de necrosis y hemorragia tumoral, irregularmente distribuidas (Figs. 2, 3 y 4). La actividad mitótica es muy baja, habiendo encontrado, tras la seriación de múltiples cortes, tan solo 1 mitosis x 50 campos de gran aumento (cga).

En la Figura 5 podemos observar la expresión positiva de actina HHF. Su inmunofenotipo podemos observarlo en la Tabla 4.

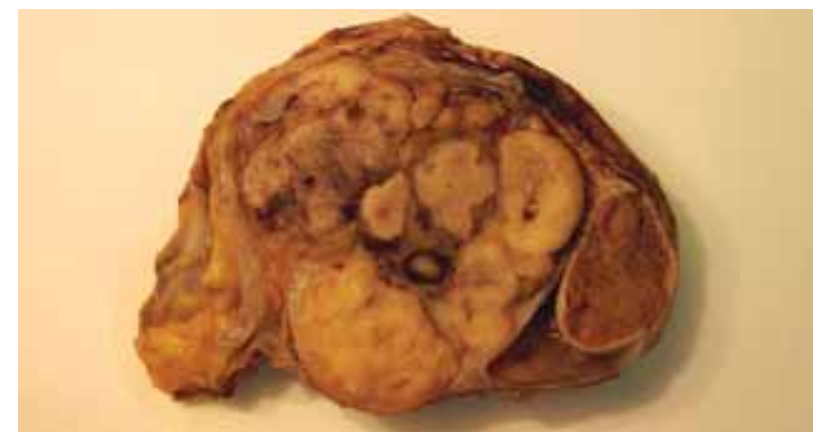

FIGURA 1. Pieza de orquiectomia -corte sagital-. Separación neta del LMS del parénquima testicular.

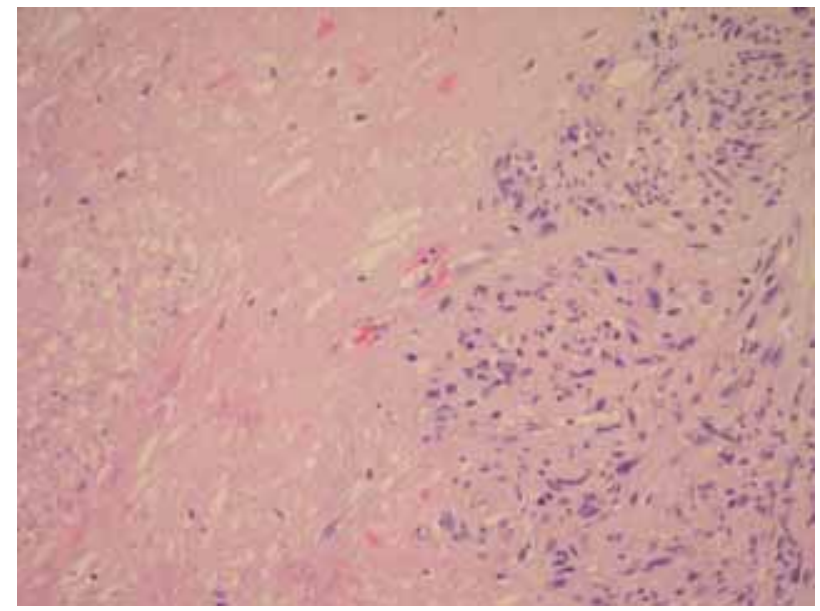

FIGURA 2. Imagen histológica (HE $x$ 200) del LMS en el área de colisión del tumor con la necrosis tumoral.

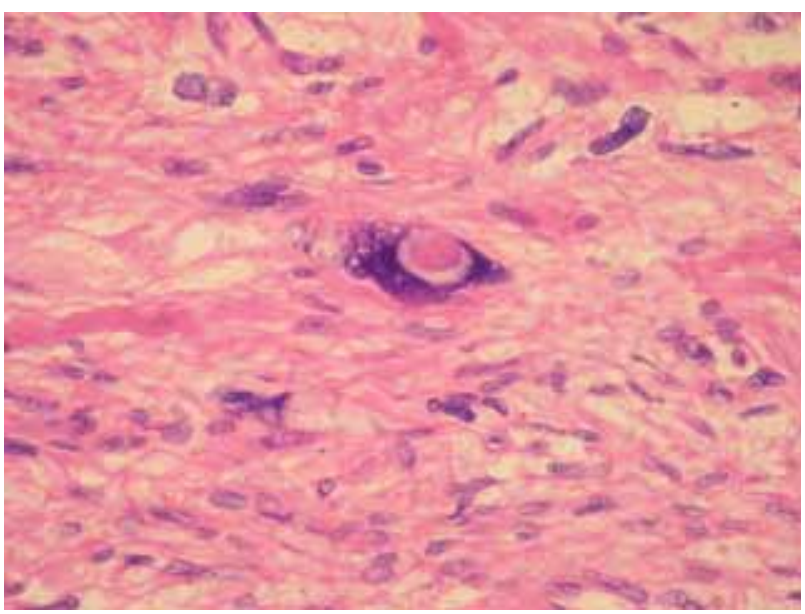

FIGURA 3. Imagen histológica (HE $x$ 400) del LMS en las zonas de pleomorfismo y dismorfismo nuclear, con inclusiones eosinófilas.

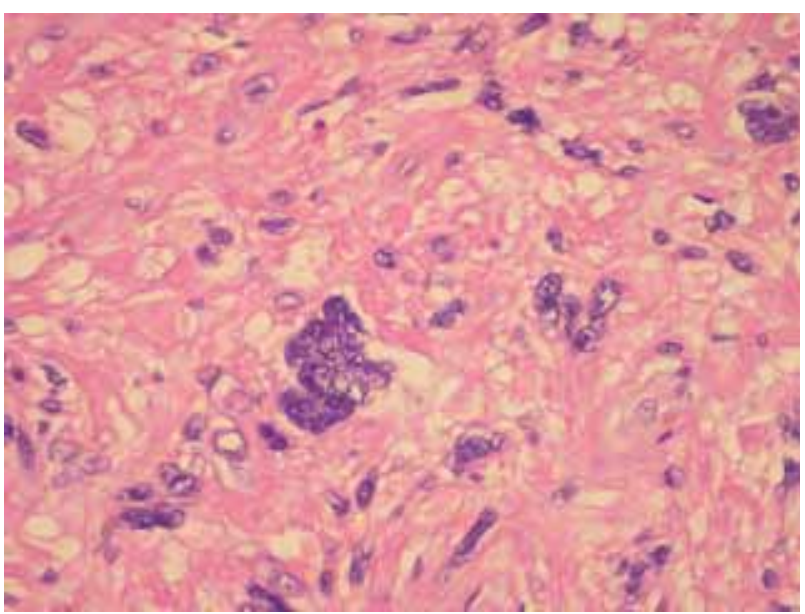

FIGURA 4. Imagen histológica (HE $x$ 400) del LMS con atipia nuclear en racimo (multinucleación).

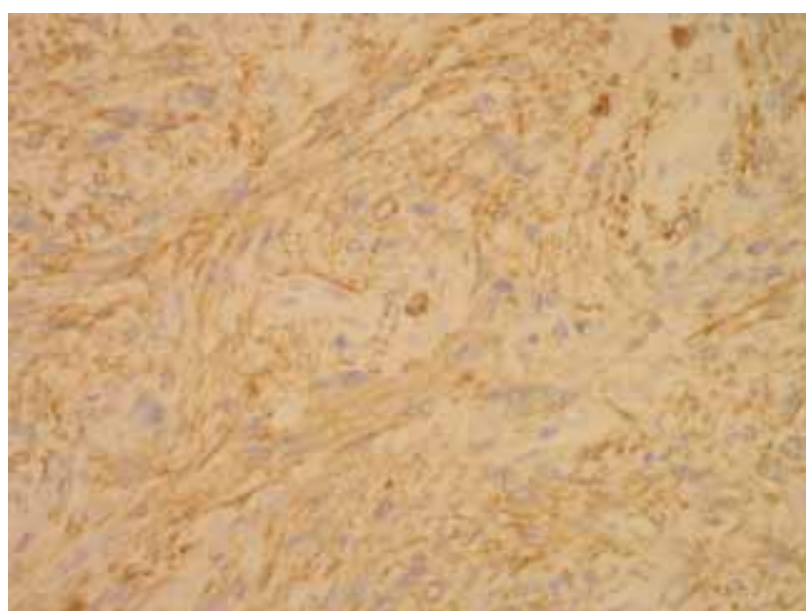

FIGURA 5. Imagen histológica del LMS con técnica de Actina HHF (Inmunohistoquímica $x$ 200)con expresión positiva de la totalidad de las células. 
Tabla 4. Panel inmunohistoquímico del caso

\begin{tabular}{lll}
\hline Marcador & \multicolumn{2}{l}{ LMS de larga evolución/caso } \\
\hline ACTINA 1A4 & Expresión Positiva & $100 \%$ \\
ACTINA HHFA & Expresión Positiva & $100 \%$ \\
DESMINA & Expresión Positiva & $100 \%$ \\
EMA & Expresión Positiva & $100 \%$ \\
VIMENTINA & Expresión Positiva & $100 \%$ \\
S-100 & Expresión Positiva & $50 \%$ \\
CD-68 & Expresión Positiva & $<12 \%$ \\
Ki-67 & Expresión Positiva & $5 \%$ \\
p-53 & Expresión Positiva & $3 \%$ \\
AE1-AE3 & Expresión Negativa \\
C KIT (C 117) & Expresión Negativa \\
Factor XIII A & Expresión Negativa \\
\hline
\end{tabular}

El diagnóstico se corresponde con un LMS Fascicular de Bajo grado (grado 1 de French) con bordes quirúrgicos libres de neoformación.

\section{DISCUSIÓN}

Presentamos un caso singular de LMS paratesticular por varios motivos. En primer lugar por su tiempo de evolución (al menos 30 meses), siendo uno de los de mayor curso clínico indolente publicados en la literatura. Tan solo hemos encontrado 1 caso anterior, con mayor tiempo de evolución (48 meses) en un varón de 33 años ${ }^{34}$. Este importante dato, contrastado en nuestro caso por la historia clínica de Atención Primaria en que se hace constar este hecho, sin que el paciente tome la decisión de acudir a atención especializada, traduce el lento proceso de este tipo de sarcomas que se corresponde en la histopatología con la buena diferenciación del mismo.

A ello sumamos una práctica ausencia de actividad mitótica en el tumor ( 1 x 50 cga) lo que haría plantearse el DD con un leiomioma celular ${ }^{21,95}$, si no fuera por las amplias áreas de necrosis, polimorfismo y atipia celular que posee, aunque con inmunofenotipo típico de la tumoración.
Aunque no existe un protocolo diagnóstico para este tipo de LMSs paratesticulares, por su baja incidencia, hemos considerado en su ausencia, aplicar los criterios de los leiomiosarcomas retroperitoneales y de tejidos blandos profundos (Tabla 5) ${ }^{93,95}$.

La amplia necrosis tumoral hallada en nuestro caso unida al polimorfismo núcleo-citoplásmico y la atipia nuclear, a pesar del bajo índice mitótico, así lo confirman.

Este hecho puede encuadrarse dentro de la teoría citogenética de que este tipo de sarcomas provienen de una transformación maligna de un tumor leiomiomatoso benigno preexistente ${ }^{63}$.

En nuestro caso lo diagnosticamos como paratesticular dado que no hemos podido filiar ni macro ni microscópicamente su origen en el cordón espermático ni en ninguna otra estructura específica de los anejos testiculares.

En cuanto al perfil inmunohistoquímico, es evidente y así lo hemos testado que su expresión está basada en la positividad de sus marcadores.

Estudios prospectivos randomizados son muy difíciles de hacer, debido a la rareza de los casos en esta localización.

Los tumores paratesticulares, se presentan como masas escrotales o inguino escrotales (como en nuestro caso) que pueden ser dolorosas, y en muchos casos acompañadas de hidrocele.

Desde el punto de vista anatomopatológico, es significativo el hecho de que existan muy pocos casos con contraste histológico en los 107 casos hallados en la bibliografía internacional, lo que se presta a muchos interrogantes.

El Diagnóstico Diferencial de los LMSs con otros sarcomas paratesticulares es, a veces, muy difícil, por confluir estirpes celulares muy semejantes y muchos de ellos con células gigantes multinucleadas que también presentan positividad para marcadores histiocitarios, con células dismórficas y pleomórficas que los hacen prácticamente indistinguibles, a pesar de que su inmunofenotipo precisa una amplia batería de

Tabla 5. French system sarcomal

\begin{tabular}{ccc}
\hline Score & Diferenciacion & Necrosis \\
\hline 0 & - & Mitosis $\mathbf{x}$ 10 cga. \\
1 & Recuerda al mesénquima normal adulto & $<50 \%$ \\
2 & Sarcoma con tipos histológicos diferentes & $<-9$ \\
3 & Sarcomas indiferenciados y embrionarios & $<50 \%$ \\
\hline
\end{tabular}

Grado 1: 2-3 scores $\quad$ Grado 2: $4-5$ scores

Grado 3: 6-7-8 scores 
negatividades para un diagnóstico de exclusión . Los estudios de extensión incluyen radiografía simple de tórax, ecografía abdominopélvica, tomografía axial computerizada abdominal y pélvica y una resonancia magnética de la región inguino-escrotal.

La investigación, en los sarcomas del cordón espermático, ha sido difícil debido a su rareza. La literatura registró solo 212 casos de 1845 a $1978^{2,18}$. Además, ha habido falta de consenso en la estrategia quirúrgica y terapéutica. Con excepción del rabdomiosarcoma embrionario, no hay en la actualidad diferencias significativas en el pronóstico de los diferentes subtipos sarcomatosos, independientemente del grado tumoral, invasión y del tamaño tumoral ${ }^{\text {99-101. }}$.

Es la ecografía el método de diagnóstico más utilizado en la evaluación diagnóstica inicial de las masas escrotales, presentando dificultades en la mayoría de los casos y obligando en la casi totalidad de los casos a una biopsia intraoperatoria, pues incluso es muy difícil precisar preoperatoriamente, si se trata de un tumor benigno o maligno. Ya hemos indicado el diagnóstico diferencial tan amplio que pueden presentar estas neoplasias antes de ser resecadas, y que solo se resuelve tras el estudio microscópico. Ante una sospecha diagnóstica, la cirugía debe ser radical

La AJCC American Joint Committee on Cancer diseñó un protocolo de estadiaje para los sarcomas de esta localización, en el que se valora el tipo histológico, el grado tumoral (G1,G2), el tamaño $(>/<5$ $\mathrm{cm}$.), la profundidad (superficial o profunda) y la presencia de metástasis ganglionares o a distancia. Sin embargo, los estudios más amplios y contrastados realizados con este tipo de tumores en los últimos años, consideran el subtipo histológico, la localización de la lesión y el estadio como factores pronósticos independientes, observando mayores supervivencias con significación estadística entre los liposarcomas y fibrosarcomas comparados con los FHMs y LMSs $(\mathrm{p}<0,001)^{86,99-101}$.

Análisis multivariantes mostraron también que la edad avanzada, el sexo masculino, las razas no caucásicas, el estadio avanzado y la localización retroperitoneal eran factores independientemente asociados con menor supervivencia, sin que el subtipo histológico fuera un factor individual pronóstico. La resección quirúrgica y la radioterapia fueron las únicas variables terapéuticas que mejoraron la supervivencia ${ }^{86}$.
La baja incidencia de este tipo de neoplasias hace además que los tratamientos no estén sistematizados y se realicen terapias individualizadas, lo que condiciona dificultad en el estudio de los resultados, generando debates y controversias. No obstante, hay algunos autores ${ }^{1}$ que consideran esta incidencia subestimada, al valorar que muchos sarcomas diagnosticados en algunas series como "sarcomas sin especificar" y sarcomas pleomórficos deberían ser reclasificados como FHMs. En una serie, en que se realizó esta reclasificación sobre un panel de 36 sarcomas paratesticulares, cambió el tipo de diagnóstico en el 35\% de los casos y el número de FHMs aumentó de 1 a $6^{9}$. Sin embargo, dado que la terapia de todo el grupo de estos sarcomas es semejante, y a pesar de la utilización de nuevos marcadores inmunohistoquímicos, el estudio reclasificatorio no ha sido una prioridad por no serlo para los oncólogos. Para la mayoría de los autores es la orquiectomía radical el tratamiento de elección y único, aunque debido a las recidivas y recurrencias que un número importante presentan, también precisan un tratamiento adyuvante. A veces es también muy dificil resecar la totalidad del tumor.

La diseminación de los sarcomas paratesticulares, generalmente, es a través del cordón espermático hacia el canal inguinal y de este a la cavidad abdominal, no encontrando casos contrastados de diseminación vascular.

\section{CONCLUSIONES}

Los LMSs paratesticulares, incluyendo los de cordón espermático, son sarcomas muy raros y de baja incidencia, tal y como hemos podido comprobar en este estudio, que precisan de una mayor convergencia diagnóstica y terapéutica de consenso entre urólogos, radiólogos, oncólogos y patólogos.

Ese hecho, señalado en todas las series, y que conduce a tratar con el mismo tipo de terapia a todos los sarcomas en esta localización (cirugía radical y radioterapia en los casos procedentes) no justifica la inexistencia de protocolos específicos para cada uno de los principales tumores mesodérmicos malignos paratesticulares (LMSs, liposarcomas, rabdomiosarcomas y FHMs). Quizás la reclasificación y el cambio diagnóstico en la revisión de algunas series realizado en los últimos años, haya servido para justificar este tipo de actitudes terapéuticas en el pasado. No obstante, consideramos que los nuevos marcadores en inmunohistoquímica y la citogenética permitirán filiar individualmente 
estas neoplasias y acceder a dianas terapéuticas específicas para estos sarcomas paratesticulares, así como los tratamientos complementarios que procedan.

La larga evolución clínica de algunos de estos sarcomas, como el nuestro, no es signo de benignidad ni de un comportamiento específico de remisión o curación. El intervalo desde la primera exploración física hasta su extirpación, y más en personas mayores, es muy amplio y no está relacionado con los grados del Sistema de French.

Por otro lado, el número de LMSs paratesticulares diagnosticados en nuestro país es también muy bajo nuestro caso es el $6^{\circ}$ descrito y aun así es importante tenerlos presentes tanto desde el punto de vista clínico, radiológico/ecográfico y oncológico como anatomopatológico.

Existen investigaciones genético-moleculares que son compatibles con la hipótesis de que un análisis comparativo entre el desequilibrio genómico más frecuente observado en las series de FHMs y de LMSs, demuestra que ambos tumores tienen recurrencias semejantes en sus desequilibrios genéticos. Al mismo tiempo, estudios de biología molecular han mostrado dianas semejantes de delecciones en sus cromosomas en ambos sarcomas. A pesar de esto, consideran los autores, que una nueva reclasificación de los sarcomas de partes blandas basado en parámetros moleculares es, en principio, todavía prematura ${ }^{19,102,103}$.

Desde estas páginas abogamos por una conferencia o reunión monográfica de las Sociedades Científicas, en las especialidades implicadas, que permitan una terapéutica específica sistematizada para cada estirpe sarcomatosa individualizada $o$ grupal, y en los subtipos que proceda, de acuerdo con las "clasificaciones reclasificadas actuales" tal y como se realiza en los sarcomas retroperitoneales y de tejidos blandos profundos.

\section{REFERENCIAS}

1. Khoubehi B, Mishra V, Ali M , Motiwala H. Karim O. Adult paratesticular tumours. BJU Internacional. 2002;90(7):707-715.

2. WHO-OMS Classification of tumours. Tumours of the urinary system and male genital organs. Eble J N, Sauter G, Epstein J I, Sesterhenn I A, Eds. IARC Press, Lyon (France). 2004 pp 273-276.

3. De Saint Aubain N. Pleomorphic cell tumors of soft tissue. Differential diagnosis. Forum of Pathology. Forpath Slide Seminar. January 2004.

4. Dotan ZA, Tal R, Golijanin D, Snyder ME, Antonescu C, Brennan MF, Russo P. Adult genitourinary sarcoma: the 25-year Memorial Sloan-Kettering experience. J Urol. 2006;176(5):2038-2039.

5. Soosay G N, Parkinson MC, Paradinas J, Fisher C. Paratesticular sarcomas: a review of cases in the British Testicular Tumour Panel and Registry. BJU Int. 1996;77(1):143-146.
6. Sane SY, Nimbkar SA, Bapat RD. Leiomyosarcoma of hydrocele sac (a case report). J Postgrad Med. 1980;26(2):145-146.

7. Gutierrez JC, Perez EA, Franceschi D, Moffat FL Jr, Livingstone AS, Koniaris LG. Outcomes for soft-tissue sarcoma in 8249 cases from a large state cancer registry. J Surg Res. 2007;141(1):105114.

8. Coindre JM. Immunohistochemistry in the diagnosis of soft tissue tumours. Histopathology. 2003;43(1):1-16.

9. Farshid G, Pradhan M, Goldblum J, Weiss SW. Leiomiosarcoma of somatic soft tissues. Am J Surg Pathol. 2002:26(1):14-24.

10. Gerber S, Futterlieb A, Schmid HP, Weimann R, Glattli A. Paratesticular sarcomas. Case report of leiomiosarcoma. Chirurg. 2000;71(8):966-968.

11. Coleman J, Brennan M F, Alektiar K, Russo P. Adult spermatic cord sarcomas: management and results. Ann Surg Oncol. 2003; 10(6):669-675

12. Woodward PJ, Schwab CM, Sesterhenn IA. Extratesticular scrotal masses:radiologic-pathologic correlation. From the archives of the AFIP. Radiographics. 2003;23(1):215-240

13. Fisher C, Goldblum JR, Epstein JI, Montgomery E. Leiomyosarcoma of the paratesticular region: A clinicopathologic study. Am J Surg Pathol. 2001;25(9):1143-1149.

14. WHO-OMS Classification of tumours. Tumours of soft tissue and bone. Fletcher CDM, Unni K, Mertens F Eds. IARC Press, Lyon (France). 2002 pp 131-134.

15. Rao CR, Srinivasulu M, Naresh KN, Doval DC, Hazarika D. Adult paratesticular sarcomas: a report of eight cases. J Surg Oncol. 1994;56(2):89-93.

16. Russo P, Brady MS, Conlon K, Hajdu SI, Fair WR, Herr HW, et al. Adult urological sarcoma. J Urol. 1992;147(4):1032-1036.

17. Lewis JJ, Leung D, Woodruff J, Brennan MF. Retroperitoneal soft-tissue sarcoma: analysis of 500 patients treated and followed at a single institution. Ann Surg. 1998;228(3):355-365.

18. Ulbright Th M, Amin MB, Young R H. Tumors of the testis, adnexa, spermatic cord and scrotum. Atlas of Tumor Pathology. Fascicle 25, 3th series. Washington, DC 1999 pp 352-353.

19. Ballo MT, Zagars GK, Pisters PW, Feig BW, Patel SR, von Eschenbach AC.. Spermatic cord sarcoma: outcome, patterns of failure and management. J Urol. 2001;166(4):1306-1310.

20. Ruiz Liso J M, Ruiz García J, del Agua Arias C, Vaillo Vinagre A, Gutiérrez Martín A, García Pérez M A. Proliferación nodular y difusa fibrosa de la túnica vaginal del testículo. 2004; 37(1):9198.

21. Billings SD, Folpe AL, Weiss SW. Do leiomyomas of deep soft tissue exist?. Am J Surg Pathol. 2001;25(9):1134-1142.

22. Goodwin WE. Multiple, bening fibrous tumors of tunica vaginalis testis. J Urol. 1946; 56: 438-447.

23. McClellan DS, Roscher A. Intrascrotal tumors in the older male. Int Surg. 1986;71(1):51-52

24. Kempson R L, Fletcher C D M, Evans H, Hendrickson M R, Sibley R K. Tumors of the soft tissues. Atlas of Tumor Pathology. Fascicle 30, 3th. series. Washington, DC 2001. pp 246-253.

25. Sternberg SS, Antonioli DA, Carter D, Mills SE, Oberman HA. (Eds.) Diagnostic Surgical Pathology, Vol 2, 3th ed., Lippincott Williams \& Wilkins. Philadelphia, 1999.

26. Braeckman J, Van Tichelen P, Dervaux G. Unusual mass of the spermatic cord. J Urol. 2003;169(6):2298-2302.

27. Jayaram N, Ramaprasad AV, Chethan M, Sujay RP. Tumours and tumour-like conditions of the paratesticular region. A study of morphological features. Indian J Pathol Microbiol. 1998;41(3): 287295.

28. Weiss SW, Goldblum JR: Enzinger and Weiss. Soft Tissues Tumors. 4th edition. Mosby. 2001.

29. Frank I, Takahashi S, Tsukamoto T, Lieber MM. Genitourinary sarcomas and carcinosarcomas in adults. In: Vogelzang NJ, Shipley WU, Scardino PT, Coffey DS, eds. Comprehensive Textbook of Genitourinary Oncology. 2nd ed. Philadelphia: Lippincott Williams \& Wilkins, 2000: 1110-1113. 
30. Berkmen F, Celebio?lu AS. Adult genitourinary sarcomas: a report of seventeen cases and review of the literature. J Exp Clin Cancer Res. 1997;16(1):45-48.

31. Fagundes M A, Zietman AL, Althausen AF, Coen JJ, Shipley WU. The management of spermatic cord sarcoma. Cancer. 1996;77(9): 1873-1876.

32. Merimsky O, Terrier P, Bonvalot S, Le Pechoux C, Delord JP, Le Cesne A. Spermatic cord sarcoma in adults. Acta Oncol. 1999;38 (5):635-638.

33. Catton CN, Cummings BJ, Fornasier V, O'Sullivan B, Quirt I, Warr D. Adult paratesticular sarcomas: a review of 21 cases. J Urol. 1991;146(2):342-345.

34. Ishii N, Yoshida S, Yoshinaga A, Ohno R, Terao T, Watanabe T, et al. Primary leiomyosarcoma of the spermatic cord with an unusually indolent clinical course. Hinyokika Kiyo. 2006;52(2): 159-161.

35. Llarena Ibarguren R, Azurmendi Sastre V, Martín Bazaco J, Villafruela Mateos A, Eizaguirre Zarza B, Pertusa Peña C. Leiomyosarcoma paratesticular. Revisión y puesta al día. Arch Esp Urol. 2004;57(5):525-530.

35. Llarena Ibarguren R, Azurmendi Sastre V, Martín Bazaco J, Villafruela Mateos A, Eizaguirre Zarza B, Pertusa Peña C. Paratesticular leiomyosarcoma. Review and update. Arch Esp Urol. 2004;57(5):525-530.

36. Masson JC, Varini JP, Masson J, Naman H, Broquerie JL. Sarcoma of the spermatic cord. Report of 2 cases. Review of the literature. Prog Urol. 1992;2(2):272-281.

37. Cruz Guerra NA, Clemente Ramos L, Montáns Araújo J, Linares Quevedo A, Pozo Mengual B, Allona Almagro A. Leiomyosarcoma paratesticular. Descripción de un Nuevo caso. Arch Esp Urol. 2002;55(3):311-314.

37. Cruz Guerra NA, Clemente Ramos L, Montáns Araújo J, Linares Quevedo A, Pozo Mengual B, Allona Almagro A. Paratesticular leiomyosarcoma: report of a new case. Arch Esp Urol. 2002;55(3): 311-314

38. Jo EJ, Kang SJ, Han CS, Shin SJ, Choi SH. A case of leiomyosarcoma of the spermatic cord. Korean J Urol. 1989;30(2):274276 (Medline).

39. Makino T, Hosono T, Tanaka T, Kuratsukuri K, Kawashima H, Nakatani T. Two cases of spermatic cord leiomiosarcoma. Hinyokika Kiyo. 2006;52(11):895-897.

40. Fall PA, Dangou JM, Diao B, Ndoye AK, Sylla C, Gueye SM, et al Leiomyosarcoma of the spermatic cord. Prog Urol. 2006 16(3):390-393

41. Inagaki T, Ebisuno S, Nagareda T. Malignant mesenchymoma of the spermatic cord with a brief review of the literature. Int J Urol. 1997;4(2):225-228

42. Lopes RI, Leite KR, Lopes RN. Paratesticular leiomyosarcoma treated by enucleation. Int Braz J Urol. 2006;32(1):66-67.

43. Takizawa A, Miura T, Fujinami K, Kawakami S, Osada Y, Kameda Y. Primary testicular leiomyosarcoma. Intern J Urol. 2005;12(6): 596-598.

44. Yoshida S, Kawakami S, Komai Y, Tadokoro M, Yokoyama M, Saito K, et al. A case of primary leiomyosarcoma of the spermatic cord. Nippon Hinyokika Gakkai Zasshi. 2004;95(5):718-721.

45. Alberghini M, Zanella L, Bacchini P, Maltarello MC, Maraldi NM, Bertoni F. Leiomyosarcoma of the spermatic cord: a light and ultrastructural description of one case. Pathol Res Pract. 2004; 200(6):487-491.

46. Secil M, Kefi A, Gulbahar F, Aslan G, Tuna B, Yorukoglu K. Sonographic features of spermatic cord leiomyosarcoma. J Ultrasound Med. 2004;23(7):973-976.

47. Ptochos A, Iosifidis N, Papazafiriou G, Kehagia-Koutoufari T, Karagiannopoulou G. Primary paratesticular epithelioid leiomyosarcoma. Urol Int. 2003;70(4):321-323.

48. Kawanishi H, Yoshida T, Kouhei N, Sasaki M, Itoh T, Yamamoto M. Leiomyosarcoma of the spermatic cord: a case report. Hinyokika Kiyo. 2003;49(9):551-554.
49. Kaneko M, Koike H, Morishita H, Emura I. A case of lipoleiomyosarcoma of the spermatic cord. Hinyokika Kiyo. 2003; 49(6):337339.

50. Ali Y, Kehinde EO, Makar R, Al-Awadi KA, Anim JT. Leiomyosarcoma complicating chronic inflammation of the testis. Med Princ Pract. 2002;11(3):157-160

51. Yoshii T, Ashizawa Y, Hariu K, Satoh M, Okana Y, Kamiyama Y, et al. A case of leiomyosarcoma of the spermatic cord. Hinyokika Kiyo. 2002;48(4):225-227.

52. Varzaneh FE, Verghese M, Shmookler BM. Paratesticular leiomyosarcoma in an elderly man. Urology. 2002;60(6):1112.

53. Bajaj P, Agarwal K, Niveditha SR, Pathania OP. Leiomyosarcoma arising from tunica vaginalis testis: a case report. Indian $J$ Pathol Microbiol. 2001;44(2):145-146.

54. Forrest M, Ahlering T. Bilateral asynchronous paratesticular leiomyosarcomas. J Urol. 2001;166(6):2309-2310.

55. Watanabe J, Soma T, Kawa G, Hida S, Koisi M. Leiomyosarcoma of the spermatic cord. Int J Urol. 1999;6(10):536-538.

56. Bertelsen CA, Jensen FS. Leiomyosarcoma of the spermatic cord. Ugeskr Laeger. 1996;158(26):3789-3790.

57. Stein A, Kaplun A, Sova Y, Zivan I, Laver B, Lurie M, et al. Leiomyosarcoma of the spermatic cord: report of two cases and review of the literature. World J Urol. 1996;14(1):59-61.

58. Ozeki S, Yasuda M, Nakano M, Ishihara S, Deguchi T, Ban Y, et al. Leiomyosarcoma of the scrotum: a case report. Hinyokika Kiyo. 1996;42(3):229-231.

59. Wiebel T, Tauber R, Schulz-Bischof K. Leiomyosarcoma of the spermatic cord. A case report with review of the literature. Urologe A. 1996;35(1):54-56.

60. Bodo G, Casetta G, Ghabin H, Vottero M, Gamba P. Leiomyosarcoma of the spermatic cord. Case report and revision of the literature. Minerva Urol Nefrol. 1994;46(3):179-181.

61. López Aramburu MA, Arroyo Muñoz JL, Velasco Oses A, Solares Cambres J. A case of leiomyosarcoma of the spermatic cord. Arch Esp Urol. 1994;47(5):521-523.

62. Gerber S, Futterlieb A, Schmid HP, Weimann R, Glättli A. Paratesticular sarcomas: case report of a leiomyosarcoma. Chirurg.. 2000;71(8):966-968.

63. Avila Padilla J, Capell González M, Muniesa Calderó M, Yelletisch Araño A, Badía Torroella F, Abós Fanlo P. Leiomyosarcoma of the spermatic cord. Actas Urol Esp. 1993;17(7):464-467.

64. Sarma DP. Leiomyosarcoma of the spermatic cord. J La State Med Soc. 1993;145(3):83-84.

65. Donovan MG, Fitzpatrick JM, Gaffney EF, West AB. Paratesticular leiomyosarcoma. Br J Urol. 1987;60(6):590.

66. Staples JJ, Pope AJ, Yoong AK. Paratesticular leiomyosarcoma. Report of two new cases and literature review. Acta Oncol. 1987;26(5):389-390.

67. Konety BR, Singh J, Lyne JC, Salud RR. Leiomyosarcoma with osteoclast-like giant cells of the spermatic cord. A case report and review of the literature. Urol Int. 1996;56(4):259-262.

68. Giménez Bascuñana A, Sola Pérez J, Server Pastor G. Giant cell leiomyosarcoma of the spermatic cord: a report. Actas Urol Esp. 1995; 19(6):476-480

69. Suarez M, Arbesú A, Ferrer L, Cotrina A, Cuervo R, Mera A. Leiomiosarcoma del cordon espermático. Rev Cub Med Mil. 2005; 34(1):19-21.

70. Singh R, Chandra A, O'Brien TS. Primary intratesticular leiomyosarcoma in a mixed race man: a case report. $\mathrm{J}$ Clin Pathol. 2004:57(12):1319-1320.

71. Bakri SJ, Krohel GB, Peters GB, Farber MG. Spermatic cord leiomyosarcoma metastatic to the orbit. Am J Ophthalmol. 2003; 136(1):213-215

72. Gillner J, Kirchberg K, Korge B, Hunzelmann N, Krieg Th. Scharffetter-Kochanek K. Cutaneous metastases from a leiomyosarcoma of the testicular tunica albugina. Hautarzt. 2000; 51(1): $41-45$ 
73. Chandra A, Baruah RK, Ramanujam, Rajalakshmi KR, Sagar G, Vishwanathan P, Raman. Primary intratesticular sarcoma. Indian J Med Sci. 2001;55(8):421-428.

74. Nagae H, Suzuki K, Fujita K. A case of leiomyosarcoma of the testis Hinyokika Kiyo. 1998 Dec;44(12):905-906.

75. Takizawa A, Miura T, Fujinami K, Kawakami S, Osada Y, Kameda Y. Primary testicular leiomyosarcoma. Intern. J. Urol 2005; 12 : 596-598.

76. Planz B, Brunner K, Kalem T, Schlick RW, Kind M. Primary leiomiosarcoma of the epididymis and late recurrence on the penis. J Urol 1998; 159(2): 508-512.

77. Diz MR, Virseda M, Ramirez JR, Merino E, Moreno A Paños P. Leiomiosarcoma escrotal con metástasis óseas. Actas Urol Esp 2006; 30(6):638-640

78. Gohji K, Oka Y, Higuchi A, Ueno K, Fujii A. Paratesticular leiomyosarcoma with elevated serum basic fetoprotein: a case report. Hinyokika Kiyo. 1993 Sep;39(9):869-872.

79. Seidl C, Lippert C, Grouls V, Jellinghaus W. Leiomyosarcoma of the spermatic cord with paraneoplastic beta-hCG production. Pathologe. 1998 Feb;19(2):146-150.

80. Rubin BP, Fletcher CD. Myxoid leiomyosarcoma of soft tissue, an underrecognized variant. Am J Surg Pathol. 2000 Jul;24(7):927936.

81. Folpe AL, Weiss SW. Lipoleiomyosarcoma (well-differentiated liposarcoma with leiomyosarcomatous differentiation): a clinicopathologic study of nine cases including one with dedifferentiation. Am J Surg Pathol. 2002 Jun;26(6):742-749.

82. Paul R, Leyh H, Hillemanns M, Hofler H, Hartung R. Giant malignant mesenchymoma of the spermatic cord with bidirectional differentiation. Onkologie. $2001 \mathrm{Feb} ; 24(1): 73-75$

83. Ou, S-M - Lee, S-S - Peng, Y-J - Sheu, L-F - Yao, N-S - Chang, SY. Production of Beta-HCG by Spermatic Cord Leiomyosarcoma: A Paraneoplastic Syndrome? J Androl. 2006;27(5):643-649.

84. Oda Y, Miyajima K, Kawaguchi K, Tamiya S, Oshiro Y. Pleomorphic leiomiosarcoma: clinicopathologic and immunohistochemical study with special emphasis on its distinction from ordinary leiomyosarcoma and malignant fibrous histyocitoma. Am J Surg Pathol. 2001;25(8):1030-1038.

85. Miettinem M. Immunohistochemistry of solid tumors. Brief review of selected problems. APMIS 1990;98(3):191-199.

86. Mondaini N, Palli D, Saieva C, Nesi G, Franchi A, Ponchietti R, et al. Clinical characteristics and overall survival in genitourinary sarcomas treated with curative intent: a multicenter study. Eur Urol. 2005;47(4):468-473.

87. Lin JS. Diagnostic application of immunohistochemistry in pleomorphic sarcomas. Zhonghua Bing Li Xue Za Zhi. 1990;19(1):6770.

88. Potti A, Ganti AK, Foster H, Knox S, Hebert BJ, Tendulkar K, et al. Immunohistochemical detection of HER-2/neu, c-kit (CD117) and vascular endothelial growth factor (VEGF) overexpression in soft tissue sarcomas. Anticancer Res. 2004;24(1):333-337.

89. Shah H, Pervez S. Immunophenotypic characterization of high grade pleomorphic sarcomas: a demographic and immunohistochemical study in a major referral center of Pakistan. J Pak Med Assoc. 2005;55(3):101-104.
90. Lai FM, Allen PW, Chan LW, Chan PS, Cooper JE, Mackenzie TM. Aggressive fibromatosis of the spermatic cord. A typical lesion in a "new" location. Am J Clin Pathol. 1995;104(4):403-407.

91. Taubert H, Würl P, Meye A, Berger D, Thamm B, Neumann K, et al. Molecular and immunohistochemical p-53 status in liposarcoma and malignant fibrous histiocytoma: identification of seven new mutations for soft tissues sarcomas. Cancer. 1995;76(7): 1187-1196.

92. Yang $P$, Hirose $T$, Hasegawa $T$, Seki $K$, Sano $T$, Hizawa $K$. Prognostic implication of the p53 protein and Ki-67 antigen immunohistochemistry in malignant fibrous histiocytoma. Cancer. 1999;76(4):618-625.

93. Ahlén J, Weng WW, Brosjö O, Von Rosen A, Larsson O, Larsson C. Evaluation of immunohistochemical parameters as prognostic markers in malignant fibrous histiocytoma. Oncol Rep. 2003;10(5) 1641-1645.

94. Baneth V, Raica M, Cimpean AM. Assessment of angiogenesis in soft-tissue tumors. Rom J Morphol Embryol. 2005;46(4):323-327.

95. Hasegawa T, Yamamoto S, Nojima T, Hirose T, Nikaido T, Yamashiro K, et al. Validity and reproducibility of histologic diagnosis and grading for adult soft-tissue sarcomas. Hum Pathol. 2002; 33(1): $111-115$.

96. Lott S, Lopez-Beltran A, Montironi R, MacLennan GT, Cheng L. Soft tissue of the urinary bladder. Part II: malignant neoplasms. Hum Pathol. 2007,38(7):963-977.

97. Winstanley AM, Landon G, Berney D, Minhas S, Fisher C, Parkinson MC. The immunohistochemical profile of malignant mesotheliomas of the tunica vaginalis. A study of 20 cases. Am J Surg Pathol. 2006;30(1):1-6.

98. Ersoz C, Aydin Ö, Gonlüsen G, Zeren H, Satar N. Light microscopic, Immunohistochemical and ultrastructural evaluation in a case of paratesticular leiomyosarcoma. J Islamic Academy Sciences 1994;7(1):61-64.

99. Lewis JJ, Leung D, Espat J, Woodruff J, Brennan MF. Effect of reresection in extremity soft tissue sarcoma. Ann Surg. 2000; 231(5):655-663.

100. Enoch S, Wharton SM, Murray DS. Management of leiomyosarcomas of the spermatic cord: the role of reconstructive surgery. World J. Surg. Oncol. 2005;3(1):23.

101. Blitzer PH, Dosoretz DE, Proppe KH, Shipley WU. Treatment of malignant tumors of the spermatic cord: a study of 10 cases and a review of the literature. J Urol. 1981;126(5):611-614.

102. Lagacé R, Aurias A. Does malignant fibrous histiocytoma exist?. Ann Pathol. 2002;22(1):29-34.

103. Tos AP. Classification of pleomorphic sarcomas: where are we now?. Histopathology. 2006;48(1):51-62.

Correspondencia autor: Dr. J.M. Ruiz Liso

Complejo Hospitalario de Soria. Hospital Virgen del Mirón

Servicio de Patología/Servicio de Urología.

Ctra. de Logroño, s/n-42071 Soria. Tel.: 975220850

E-mail autor: jruiz@hsor.sacyl.es

Información artículo: Original - Revisión

Trabajo recibido: septiembre 2007

Trabajo aceptado: abril 2008 